\title{
Diagnosis and Management of a Large Adrenal Myelolipoma
}

\author{
Imad Ghantous ${ }^{1}$ Melissa Kyriakos Saad ${ }^{2,0}$ Toufic Saber ${ }^{2} \quad$ Elissa Mahfouz ${ }^{2} \quad$ Imad Matta ${ }^{1}$ \\ Elias Saikaly²,
}

${ }^{1}$ Department of Urology, Saint George Hospital University Medical Center, University of Balamand, Beirut, Lebanon

2Department of General Surgery, Saint George Hospital University Medical Center, University of Balamand, Beirut, Lebanon

\begin{abstract}
Address for correspondence Elias Saikaly, MD, Department of General Surgery, Saint George Hospital University Medical Center, University of Balamand, Beirut 961, Lebanon (e-mail: dreliassaikaly@gmail.com).
\end{abstract}

Int J Recent Surg Med Sci:2020;6:70-73

\begin{abstract}
Keywords

- adrenal myelolipoma

- CT scan

- biopsy

- bilateral

Adrenal myelolipoma is a rare condition. Although benign in nature, these tumors can increase in size and cause a mass effect to nearby structures presenting as abdominal distension and pain. In 90\% of cases, adrenal myelolipomas are unilateral. However, the real challenge in management is when bilateral adrenal myelolipoma is present. Herein, we present a case of a 51-year-old male Caucasian patient with bilateral adrenal myelolipoma. The patient had a large left adrenal myelolipoma $(23 \times 13 \mathrm{~cm})$ and a small one on the right $(4.4 \times 4 \mathrm{~cm})$. We opted for an open left adrenalectomy due to classical features on computed tomography scan and the mass effect of the tumor.
\end{abstract}

\section{Introduction}

Adrenal myelolipoma was first described by Gierke in 1905; it is a benign tumor that tends to grow asymptomatically up until a mass effect starts to cause symptoms. ${ }^{1}$ Recently, there has been an increase in the numbers of reported cases due to the increasing frequency in utilizing the use of imaging modalities in investigation of abdominal pain leading to an incidence ranging between 0.08 and $0.4 \% .^{1-3}$ These tumors constitute 3 to $5 \%$ of all adrenal tumors on autopsies. ${ }^{1,2}$ Prior to year 2000, less than 300 cases were reported, with the majority of cases being unilateral. ${ }^{3}$ Bilateral myelolipomas are found in up to $10 \%$ of the cases. ${ }^{4}$ Adrenal myelolipomas affect both genders equally. The largest reported tumor weighed $6 \mathrm{~kg}$ and measured $31 \times 24.5 \times 11.5 \mathrm{~cm}$. ${ }^{5,6}$ These tumors are composed of fat and hematopoietic tissue and are nonsecretory in most cases. ${ }^{6}$ It is important to mention that these tumors have no malignant potential.,4

The management of adrenal myelolipoma is controversial and challenging, especially in the cases where bilateral adrenal myelolipomas are present. ${ }^{7}$ However, decision for preoperative biopsy poses another challenge in the management

published online

December 2, 2020
DOI https://doi.org/ 10.1055/s-0040-1721519 ISSN 2455-7420. due to the presence of liposarcoma as a differential diagnosis based on imaging characteristic and the potential of postbiopsy hemorrhage. ${ }^{4}$

Herein, we present a case of 51-year-old Caucasian male patient presenting with bilateral adrenal myelolipoma.

\section{Case Presentation}

This is the case of a 51-year-old male patient with a history of cerebrovascular accident since 2011 with no permanent sequella, hypertension treated with a combination of angiotensin II receptor blocker and hydrochlorothiazide, and untreated dyslipidemia presenting for abdominal bloating and diffuse, postprandial pain along with early satiety. The patient is a social smoker and alcohol drinker with no record of previous abdominal surgery. He started experiencing these symptoms 3 years prior to presentation, and 2 months prior to presentation symptoms increased in severity. On physical exam, abdominal distension with tenderness on the left side was observed. His laboratory tests came back within the normal range. A colonoscopy and gastroscopy scheduled and were normal, but revealed a mass effect on the descending colon 
suggestive of an extracolonic compression. The patient was referred to radiological imaging. Contrast-enhanced computed tomography (CT) scan showed a large well-circumscribed lesion, measuring $13 \times 23 \mathrm{~cm}$ in the largest anteroposterior and transverse dimensions, containing fat, stellate calcifications, and soft tissue components, likely emerging from the left adrenal gland. In addition to that, a subsequent mass effect with displacement of bowels, spleen, stomach, and pancreas without obstruction, as well as slight compression on the left renal vein and left kidney, were noted. On the right side, a smaller $4.8 \times 4.4 \mathrm{~cm}$ well-circumscribed right adrenal lesion containing fat and soft tissue components was observed ( - Fig. 1). These findings are consistent with bilateral adrenal myelolipoma ( - Fig. 2). Further laboratory tests were done to rule out functional tumor of the adrenal gland that came back normal, thus prompting the diagnosis of non-secretory adrenal myelolipoma.

The patient was scheduled for an open left adrenalectomy. The tumor had a good cleavage plane all over the dissection except on the descending colon where segmental resection was opted in order not to jeopardize the treatment plan in case the final pathology result came different than the anticipated one. Macroscopically, the specimen was relatively well circumscribed, fatty with red-brown areas measuring $21 \times 16 \times 12 \mathrm{~cm}$ and weighing $2,540 \mathrm{~g}$. The definitive pathology result of the specimen turned out to be consistent with adrenal myelolipoma. There were no perioperative or postoperative complications. The patient was discharged home on postoperative day 6 with recommendation for a CT scan follow-up every 6 months on the adrenal myelolipoma found on the right side. The follow-up after 6 months showed a well-healed patient with a stable size of right adrenal myelolipoma.

\section{Discussion}

Adrenal myelolipomas are benign tumors found incidentally in most cases. ${ }^{2}$ The etiology is uncertain and many reports hypothesize that stressful stimuli and chronic use of nonsteroidal anti-inflammatory drugs trigger its growth. ${ }^{1,4}$ This condition is associated with multiple adrenal disorders such as hypertension, Cushing's disease, diabetes type II, and obesity (truncal obesity mainly). ${ }^{1}$ The majority of those tumors is relatively small with a size that rarely reaches $4 \mathrm{~cm}$, and they exhibit no endocrinologic function. ${ }^{2,8}$ No gender difference is seen among the distribution of these tumors, and they tend to occur between the fifth and seventh decade. ${ }^{2,6}$ In addition, these tumors present bilaterally in only $10 \%$ of cases. ${ }^{2}$

Adrenal myelolipoma when symptomatic presents with diffuse abdominal pain, abdominal distension, and early satiety. ${ }^{1,2}$ The abdominal pain is due to tearing on the renal pedicle causing referring visceral pain. ${ }^{8}$ Abdominal distension is due to the ability of those tumors to increase in size and cause mass effect on adjacent organs. ${ }^{2}$ Needless to mention, early satiety is a result of the space-occupying tumor that induces this effect. ${ }^{1,2}$
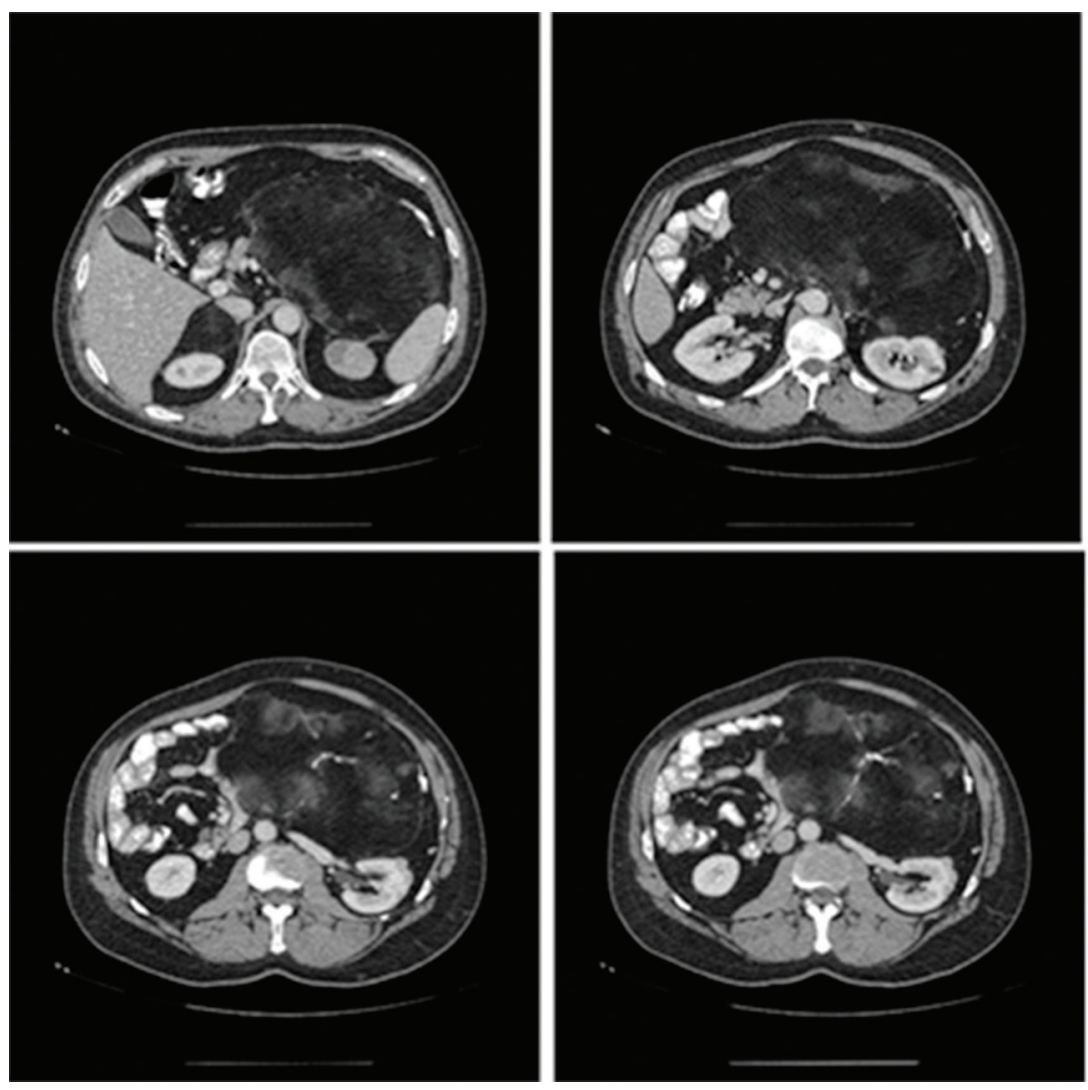

Fig. 1 Contrast-enhanced computed tomography showing adrenal tumor on both sides with the largest one on the left side. 


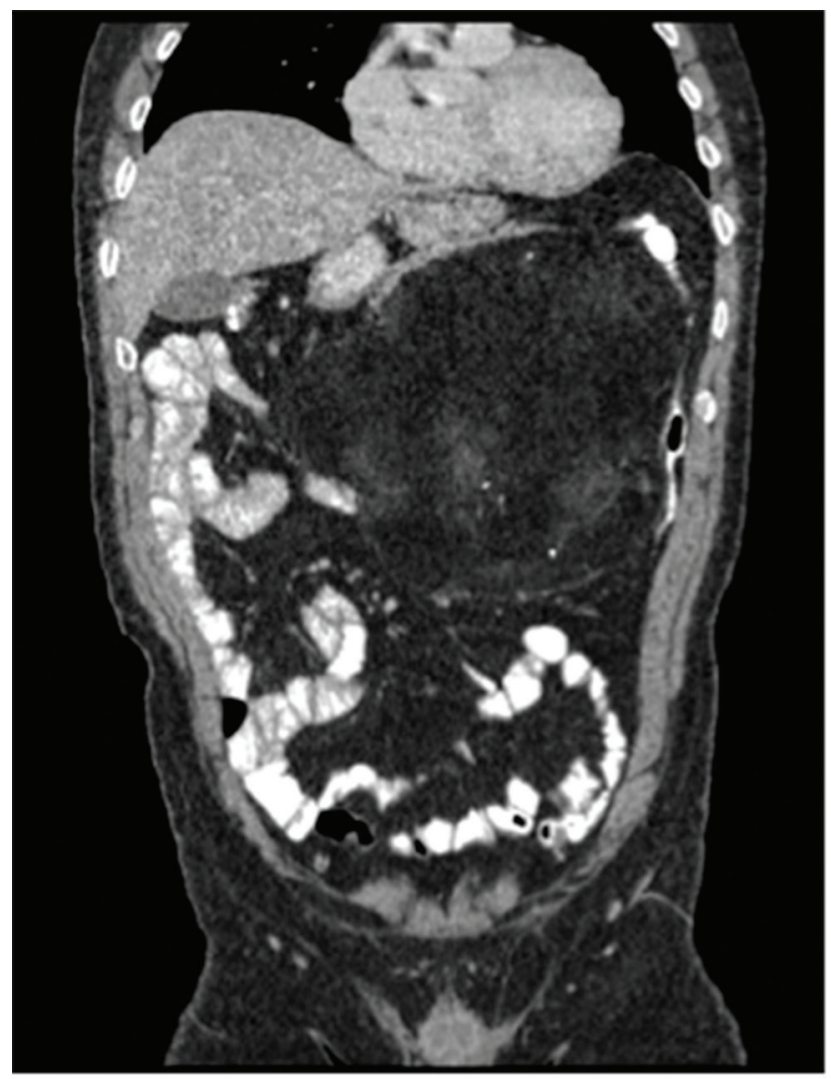

Fig. 2 Contrast-enhanced computed tomography showing large tumor on the left side causing mass effects.

The challenge is posed on how to manage a bilateral tumor on presentation. ${ }^{2,7}$ Recent reports suggest unilateral adrenalectomy for the largest tumor to avoid all the endocrinologic drawbacks. ${ }^{1,2}$ In most of the reported bilateral cases, one side is observed to have a small tumor (usually $<4 \mathrm{~cm}$ ), while the other side, having a larger tumor, causing the patient's symptoms; therefore, the removal of the latter is a good option for management if the patient is followed closely postoperatively for any change in size in the contralateral lesion. ${ }^{2}$ Recently, laparoscopic management of the tumor has shown superiority in recovery and reduced hospital stay. ${ }^{4,8}$ However, due to the large size of the tumor at presentation in numerous cases, laparoscopic management can turn out to be challenging or even inadequate. For these reasons, most of the literature recommends open surgeries for the management of tumors more than $7 \mathrm{~cm} .^{4}$

Moreover, conservative management is suggested in most of the confirmed cases of adrenal myelolipoma measuring less than $4 \mathrm{~cm} .{ }^{4}$ This management is coupled with regular imaging: ultrasound or CT to monitor the evolution of the tumor. ${ }^{4}$ This type of management is shifted to a surgical one in very specific situations: tumor growth exceeding $6 \mathrm{~cm}$, symptomatic manifestations, and life-threatening rupture of the tumor. ${ }^{3}$ Life-threatening rupture of adrenal myelolipoma is the only emergency for surgical intervention. ${ }^{4,9}$ In case of a rupture in the presence of bilateral adrenal myelolipoma, the recommendation is to remove the ruptured tumor only; moreover, open surgery is the preferred surgical methodology to be adopted. ${ }^{9}$ In addition to that, bilateral adrenalectomy should be avoided since the patient will be required to receive lifelong steroid supplements after such a procedure. ${ }^{9}$

The preoperative biopsy of adrenal myelolipoma is another challenging topic. It is important to specify that a definitive diagnosis of adrenal myelolipoma depends on the definitive pathology result of the specimen. ${ }^{4}$ Fine-needle aspiration can be used for preoperative diagnosis. ${ }^{2}$ If fine-needle biopsy is to be done preoperatively, the tract of the needle should be excised with the specimen due to an increased risk of seeding. ${ }^{2,4}$ When classical radiological features are present on imaging, performing a biopsy may not be mandatory. ${ }^{4}$

CT scan and magnetic resonance imaging (MRI) have a very high sensitivity and specificity and can harbor pathognomonic features of adrenal myelolipoma. ${ }^{4,6}$ The presence of adrenal fat is pathognomonic on CT scan for adrenal myelolipoma and is found in around 55\% of cases, along with the presence of calcification in $27 \%{ }^{6}$ In addition to that, a low Hounsfield unit between-30 and -100 is seen on $\mathrm{CT}^{6}$ suggestive of myelolipoma. On MRI findings, adrenal myelolipoma revealed high $\mathrm{T} 1$ and $\mathrm{T} 2$ signal intensities with signal loss following fat suppression. ${ }^{6}$ Absence of these imaging features allows for multiple differential diagnosis to be raised such as liposarcoma, retroperitoneal lipoma, and renal angiomyelolipoma. ${ }^{1}$ This fact renders the management of these tumors more challenging due to the risk of missing a malignant tumor when going through the differential diagnosis. ${ }^{4,6}$.

Back to our case, and based on Dieckmann et al, the tumor on the left side fits two criteria for surgical management: large size $(>7 \mathrm{~cm})$ and cause of displacement symptoms. ${ }^{10,11}$ Besides, the tumor was symptomatic. We decided on a unilateral left adrenalectomy followed by a close follow-up of the tumor on the right side to reduce the risk of adrenal insufficiency. We avoided biopsy given the tumor's classical appearance on imaging. Finally, we performed an open surgery due to the large size of the tumor measuring $23 \mathrm{~cm}$ and weighing an estimation of $2.5 \mathrm{~kg}$. Our suggested plan for follow-up was a CT scan every 6 months.

\section{Conclusion}

The majority of adrenal myelolipomas presents unilaterally and its management is surgical excision once symptomatic. $^{2}$ However, management of bilateral adrenal myelolipoma is very challenging. ${ }^{2.7}$ In most of the cases, the patient presents with pain on one specific side of the abdomen, usually corresponding to the side where the tumor has the largest size and can be attributable to traction on the renal pedicle. ${ }^{8}$ Preoperative biopsy is questionable due to the high risk of seeding in case the tumor was not a 
myelolipoma. ${ }^{4}$ Therefore, we recommend a biopsy in selected cases followed by an excision of the largest tumor by open surgery if the tumor is larger than $7 \mathrm{~cm}$. Close monitoring of the contralateral remaining tumor every 6 months using ultrasound, CT scan, or MRI should follow this step. ${ }^{4,6}$ The future decision of removal of the contralateral tumor will depend on sudden increase in size, rupture, and hemorrhage. ${ }^{3}$ Naturally, lifelong cortisol supplements will be needed to compensate for adrenal insufficiency. ${ }^{9}$

Adrenal myelolipoma is a challenging entity in the surgical field. ${ }^{7}$ The collaboration between endocrinologists, radiologists, surgeons, and oncologists is highly recommended to ensure the proper management of the patient.

\section{Note}

An informed consent was signed by the patient, authorizing access on his medical records and completion of this work.

\section{Conflict of Interest}

None declared.

\section{References}

1 Nabi J, Rafiq D, Authoy FN, Sofi GN. Incidental detection of adrenal myelolipoma: a case report and review of literature. Case Rep Urol 2013;2013:78948110.1155/2013/789481

2 Ioannidis O, Papaemmanouil S, Chatzopoulos S, et al. Giant bilateral symptomatic adrenal myelolipomas associated with congenital adrenal hyperplasia. Pathol Oncol Res 2011;17(3): 775-778

3 Ramirez M, Misra S. Adrenal myelolipoma: to operate or not? A case report and review of the literature. Int J Surg Case Rep 2014;5(8):494-496

4 Hsu SW, Shu K, Lee WC, Cheng YT, Chiang PH. Adrenal myelolipoma: a 10-year single-center experience and literature review. Kaohsiung J Med Sci 2012;28(7):377-382

5 Brogna A, Scalisi G, Ferrara R, Bucceri AM. Giant secreting adrenal myelolipoma in a man: a case report. J Med Case Reports 2011;5:29810.1186/1752-1947-5-298

6 Low G, Dhliwayo H, Lomas DJ. Adrenal neoplasms. Clin Radiol 2012;67(10):988-1000

7 Shenoy VG, Thota A, Shankar R, Desai MG. Adrenal myelolipoma: controversies in its management. Indian J Urol 2015; 31(2):94-101

8 Gadelkareem RA, Khalil MM, Mohammed N, Makboul R, Badary FA. Laparoscopic excision of a large symptomatic and kidney-displacing adrenal myelolipoma: a case report. Afr J Urol 2018;24(1):636

9 Zattoni D, Balzarotti R, Rosso R. The management of bilateral myelolipoma: case report and review of the literature. Int J Surg Case Rep 2015;12:31-36

10 Jia-Hwia W. Detection and treatment of adrenal myelolipomas. Urol Sci 2011;22(1):38-39

11 Dieckmann KP, Hamm B, Pickartz H, Jonas D, Bauer HW. Adrenal myelolipoma: clinical, radiologic, and histologic features. Urology 1987;29(1):1-8 